\title{
CORRIGENDUM: COMPLEXITY OF INFINITE WORDS ASSOCIATED WITH BETA-EXPANSIONS
}

\author{
Christiane Frougny ${ }^{1,2}$, ZuZana Masáková ${ }^{3}$ And \\ Edita Pelantová ${ }^{3}$
}

\begin{abstract}
We add a sufficient condition for validity of Proposition 4.10 in the paper Frougny et al. (2004). This condition is not a necessary one, it is nevertheless convenient, since anyway most of the statements in the paper Frougny et al. (2004) use it.
\end{abstract}

Mathematics Subject Classification. 11A63, 11A67, 37B10, $68 \mathrm{R} 15$

RAIRO-Inf. Theor. Appl. 38 (2004) 163-185.

\section{INTRODUCTION}

The aim of this note is to correct the mistake contained in our paper [2]. We shall use the notation of the paper and refer to the statements included in it.

We were pointed out [1] a counterexample to assertion (1) of Theorem 6.2 in the paper. The assertion says that the complexity of the fixed point $u_{\beta}$ of the canonical substitution $\varphi_{\beta}$ associated with a simple Parry number $\beta$ with the Rényi expansion $d_{\beta}(1)=t_{1} t_{2} \cdots t_{m-1} 1$ is affine, namely $\mathcal{C}(n)=(m-1) n+1$. This statement is however true only under the condition used for assertion (2) of the theorem, namely that the Rényi expansion $d_{\beta}(1)=t_{1} t_{2} \cdots t_{m}$ satisfies

$$
t_{1}=t_{2}=\cdots=t_{m-1} \quad \text { or } \quad t_{1}>\max \left\{t_{2}, \ldots, t_{m-1}\right\} .
$$

The mistake occurred due to a slip in the proof of Proposition 4.10. We show in this note that under the additional condition $(*)$ the proposition is valid.

\footnotetext{
1 LIAFA, CNRS UMR 7089, 2 place Jussieu, 75251 Paris Cedex 05, France;

e-mail: christiane.frougny@liafa.jussieu.fr

2 Université Paris 8.

3 Department of Mathematics, FNSPE, Czech Technical University, Trojanova 13, 12000 Praha 2, Czech Republic;

e-mail: masakova@km1.fjfi.cvut.cz \& pelantova@km1.fjfi.cvut.cz 
The corrected version of Proposition 4.10 of [2] is stated here as Proposition 2.2. At the end of this note we explain which statements of the paper [2] need to be equipped with condition $(*)$, as well.

Let us mention that the condition $(*)$ in Proposition 2.2 may be weakened. Nevertheless, we have chosen the condition in the form $(*)$, since anyway most of the statements in the paper [2] use it.

\section{Proof of Proposition 4.10 of [2]}

In order to prove Proposition 2.2 we need the following lemma.

Lemma 2.1. Let $t_{1}>\max \left\{t_{2}, \ldots, t_{m-1}\right\}$. Let $w$ be a right special factor of $u_{\beta}$ with at least 3 distinct right extensions $X, Y, Z$, such that $w$ contains a non-zero letter, $w X$ is a left special factor and $X \neq 0$. Then there exists a word $\tilde{w}$ which is a right special factor of $u_{\beta}$ with at least 3 distinct right extensions $\tilde{X}, \tilde{Y}, \tilde{Z}$ such that $\tilde{w} \tilde{X}$ is a left special factor, $\tilde{X} \neq 0$, and $w X=\varphi(\tilde{w} \tilde{X})$.

Proof. The word $w$ can be written as $w=w^{\prime} U 0^{p}$, where $U \neq 0$ and $p \geq 0$. Thus $U 0^{p} X, U 0^{p} Y, U 0^{p} Z$ are factors of $u_{\beta}$. Since at least one of $X, Y, Z$ is $\geq 2$, we can derive from Lemma 4.5 of [2] and condition $t_{1}>\max \left\{t_{2}, \ldots, t_{m-1}\right\}$ that $p<t_{1}$. Since $w^{\prime} U$ is a left special factor, according to (ii) of Lemma 3.7 there exists a left special factor $\tilde{w}$ such that $w^{\prime} U=\varphi(\tilde{w})$. Now

$$
\begin{aligned}
& w X=\varphi(\tilde{w}) 0^{p} X \\
& w Y=\varphi(\tilde{w}) 0^{p} Y \\
& w Z=\varphi(\tilde{w}) 0^{p} Z
\end{aligned}
$$

are distinct factors of $u_{\beta}$. Hence there must exist distinct letters $\tilde{X}, \tilde{Y}, \tilde{Z}$ such that $\tilde{w} \tilde{X}, \tilde{w} \tilde{Y}, \tilde{w} \tilde{Z}$ are also factors of $u_{\beta}$. Moreover, since $X \neq 0$ and $p<t_{1}$, we have $\varphi(\tilde{X})=0^{p} X$, where $\tilde{X} \neq 0$. As $\varphi(\tilde{w} \tilde{X})=w X$ is a left special factor, (ii) of Lemma 3.7 implies that $\tilde{w} \tilde{X}$ is a left special factor, which completes the proof.

The following statement is the same as in Proposition 4.10 of [2], except the additional condition $(*)$.

Proposition 2.2. Let $d_{\beta}(1)$ satisfies the condition $(*)$. Then for every maximal left special factor $v=v_{0} v_{1} \cdots v_{k}$ containing a letter $v_{j} \neq 0$ there exists a maximal left special factor $w$ and an $s \in\left\{t_{1}, t_{2}, \ldots, t_{m-1}\right\}$ such that $v=\varphi(w) 0^{s}$.

Proof. Let $j=\max \left\{i \mid v_{i} \neq 0\right\}$. According to Lemma 3.7 there exists a left special factor $w=w_{0} w_{1} \cdots w_{\ell}$ such that $v_{0} v_{1} \cdots v_{j}=\varphi\left(w_{0}\right) \varphi\left(w_{1}\right) \cdots \varphi\left(w_{\ell}\right)$ and thus

$$
v=v_{0} v_{1} \cdots v_{j} 0^{s}=\varphi\left(w_{0}\right) \varphi\left(w_{1}\right) \cdots \varphi\left(w_{\ell}\right) 0^{s}, \quad \text { where } s=k-j .
$$

Since $v$ is maximal, we can use Observation 4.2 and Corollary 4.6 to derive that $s \in\left\{t_{1}, t_{2}, \ldots, t_{m-1}\right\}$. 
It remains to show that $w$ is a maximal left special factor of $u_{\beta}$. Assume that $w$ is not maximal. We distinguish two cases according to which part of condition $(*)$ is satisfied.

- Let $t_{1}=t_{2}=\cdots=t_{m-1}=: t$. Since $w$ is not maximal, then according to Lemma 4.9 there exists a left special factor $w X$, where $X \neq m-1$ or a left special factor $w(m-1) 0$. However, then (ii) of Lemma 3.7 implies that $\varphi(w X)=\varphi(w) 0^{t}(X+1)$, resp. $\varphi(w(m-1) 0)=\varphi(w) 0^{t_{m}+t} 1$, is also a left special factor. Since $s=t$, the factor $v$ is a proper prefix of both of them, which is a contradiction with the maximality of $v$.

- Let $t_{1}>\max \left\{t_{2}, \ldots, t_{m-1}\right\}$. Since $v=\varphi(w) 0^{s}$ is a maximal left special factor of $u_{\beta}$ and $w$ is not maximal, there exists a letter $X$ such that $w X$ is again a left special factor. Lemma 3.7 implies that $\varphi(w X)$ is also a left special factor. Since $v=\varphi(w) 0^{s}$ may not be a proper prefix of $\varphi(w X)$, the condition $t_{1}>\max \left\{t_{2}, \ldots, t_{m-1}\right\}$ implies $X \neq 0$.

The maximality of the left special factor $v=\varphi(w) 0^{s}$ implies also existence of distinct letters $Y^{*}, Z^{*}$ such that $\varphi(w) 0^{s} Y^{*}, \varphi(w) 0^{s} Z^{*}$ are factors of $u_{\beta}$ and but they are not left special. There must exist distinct letters $Y, Z$ such that $w Y, w Z$ are factors of $u_{\beta}$ but not left special.

We have thus shown that $w$ is a right special factor with at least 3 distinct right extensions $X \neq 0, Y, Z$, where $w X$ is a left special factor. Repeated use of Lemma 2.1 leads to a right special factor $w^{(0)}=0^{q}$, for $q \geq 1$, which has at least 3 distinct right extensions $X^{(0)} \neq 0, Y^{(0)}, Z^{(0)}$, such that $w^{(0)} X^{(0)}$ is a left special factor of $u_{\beta}$. Lemma 4.5 implies that $X^{(0)}=1$ and $q=t_{1}$. At least one letter among $Y^{(0)}, Z^{(0)}$ is non-zero, say $Y^{(0)}$. Then $Y^{(0)} \geq 2$, but then $w^{(0)} Y^{(0)}=0^{t_{1}} Y^{(0)}$ is due to Lemma 4.5 not a factor of $u_{\beta}$, which is a contradiction.

\section{Conclusions}

Proposition 4.10 was used in [2] for proving Corollary 4.11, second implication of Theorem 4.12, assertion (1) of Theorem 6.2 and Corollary 6.3. Therefore condition $(*)$ should be added in the mentioned statements as well.

Acknowledgements. We are grateful to V. Berthé and J. Bernat for pointing out a counterexample to assertion (1) of Theorem 6.2 in our paper.

\section{REFERENCES}

[1] J. Bernat and V. Berthé, Oral communication.

[2] Ch. Frougny, Z. Masáková and E. Pelantová, Complexity of infinite words associated with beta-expansions. RAIRO-Inf. Theor. Appl. 38 (2004) 163-185. 